\title{
A Case of Acute Bilateral Irvine-Gass Syndrome following Uncomplicated Phacoemulsification, Demonstrated with Optical Coherence Tomography
}

\author{
Melissa K. Shields Paul A. Adler Dujon R.W. Fuzzard \\ Rajeeve Chalasani Joanne M.Y. Teong \\ Parke Street Specialist Centre, Katoomba, N.S.W., Australia
}

\section{Key Words}

Irvine-Gass syndrome $\cdot$ Cystoid macular edema, bilateral $\cdot$ Cataract surgery $\cdot$ Optical coherence tomography

\begin{abstract}
Purpose: To report a case of acute bilateral Irvine-Gass syndrome. Methods: This is an observational case report. Results: An 82-year-old man with no significant ocular history developed postsurgical pseudophakic cystoid macular edema (CME; Irvine-Gass syndrome) on consecutive phacoemulsification cataract surgeries. His initial first-eye (left) CME developed 25 days after surgery and was managed with topical preparations of dexamethasone $0.1 \%$ and ketorolac $0.4 \%$, in addition to a routine post-cataract surgery drop regime. His left CME resolved completely on optical coherence tomography (OCT) by day 100 , and he subsequently (after extensive discussion of CME risks) underwent cataract surgery on his right eye. He was commenced prophylactically on dexamethasone, ketorolac and oral indomethacin 25 mg t.d.s. immediately after surgery; however, he later developed CME (OD) on day 32 postoperatively. Within 6 months, he achieved complete resolution of his CME in both eyes. His clinical course was documented with serial OCT studies. Conclusion: Irvine-Gass syndrome remains an important differential diagnosis in the evaluation of blurred vision after cataract surgery, despite decreasing incidence. Those who experience CME following their first cataract operation should be counseled about the risks of developing the condition in the contralateral eye, despite prophylactic measures.

(c) 2015 S. Karger AG, Basel
\end{abstract}

KARGER 125is $\quad \begin{aligned} & \text { Melissa K. Shields } \\ & \text { Parke Street Specialist Centre } \\ & \text { PO Box 867 } \\ & \text { Katoomba, NSW 2780 (Australia) } \\ & \text { E-Mail melissa.kate.ellis@gmail.com }\end{aligned}$


Shields et al:: A Case of Acute Bilateral Irvine-Gass Syndrome following Uncomplicated Phacoemulsification, Demonstrated with Optical Coherence Tomography

\section{Introduction}

Cystoid macular edema (CME) following cataract surgery, also known as Irvine-Gass syndrome, is an important differential diagnosis of painless decrease in vision following uneventful cataract extraction [1]. The pathogenesis is likely multifactorial, ultimately leading to the breakdown of the blood-retinal barrier and cystic accumulation of extracellular intraretinal fluid [2].

The introduction of modern surgical techniques including phacoemulsification and small incision cataract surgery has contributed to a reduction in the incidence of CME since it was initially reported by Irvine in 1953 [3]. CME is traditionally defined as either clinical CME or angiographic CME. Angiographic CME is detected by fluorescein angiography and is significantly more common than clinical CME. Clinical and optical coherence tomography (OCT) evidence of CME after uncomplicated cataract surgery is present in around 0.1-2.35 and $5 \%$, respectively $[1,4,5]$. The diagnosis of CME was previously made with fluorescein angiography after the patient became symptomatic; however, the emergence of OCT has provided a quick and noninvasive means of diagnosis [1].

Patient factors predisposing to CME include comorbidities with high vasoactive profile (diabetic retinopathy, uveitis), while surgical factors include inflammation-inducing complications such as retained lens material, vitreous loss and vitreomacular traction [6]. However, many otherwise healthy patients who undergo routine surgery continue to develop the condition.

The most frequently applied treatment paradigms include aggressive topical nonsteroidal anti-inflammatory drugs or steroids aimed at minimizing inflammation [6, 7]. While the condition is usually self-limiting and the resolution of symptoms is likely to occur within 312 months, it may occasionally persist and lead to permanent vision loss [6]. The prevention of Irvine-Gass syndrome continues to pose challenges for ophthalmologists, due to its ability to affect individuals without any obvious predisposing factors at variable times of onset postoperatively.

\section{Case Report}

An 82-year-old male with no significant past ocular history was referred for evaluation and treatment of progressive blurred vision secondary to bilateral cataract (1+ cortical, 2+ nuclear sclerotic) by his optometrist. His best-corrected visual acuity (BCVA) was 6/9 in both eyes, with no evidence of any other ocular disease. His past medical history was significant only for hypertension, which was well controlled with oral amiodarone and hydrochlorothiazide. Preoperative OCT examination showed normal maculae bilaterally (fig. 1a, fig. 2a).

He underwent routine phacoemulsification surgery in the left eye with implantation of an intraocular lens [single-piece acrylic toric lens (SN6AT3); Alcon, Fort Worth, Tex., USA]. The patient received preoperative topical diclofenac $0.1 \%$ hourly for $4 \mathrm{~h}$ and a postoperative weaning regimen of chloramphenicol $0.5 \%$ and diclofenac $0.1 \%$ eye drops, which were ceased on day 28.

Postoperatively at day 9, BCVA decreased to $6 / 18$, with minor fluorescein staining of the cornea. OCT showed no macular edema (fig. 1b). At day 16, BCVA had improved to 6/9. Consequently, OCT was not performed. At day 22, BCVA was reduced to 6/24. OCT showed classical pseudophakic CME (fig. 1c). Ketorolac 0.4\% eye drops 6 times per day were com- 
Shields et al.: A Case of Acute Bilateral Irvine-Gass Syndrome following Uncomplicated Phacoemulsification, Demonstrated with Optical Coherence Tomography

menced, and the patient was referred for medical retina opinion, at which time $0.1 \%$ dexamethasone drops 6 times per day was commenced. OCT was used at the sole diagnostic imaging modality.

The patient responded to topical therapy without further intervention (fig. 1d) and was successfully weaned off all drops 3 months postoperatively. Visual acuity in the left eye had improved to $6 / 6$ uncorrected. The appearance of the right macula remained unchanged. Once the patient's macular edema had resolved in the left eye, and following extra risk counseling, routine cataract surgery was performed in the right eye [single piece acrylic toric lens (SN6AT4); Alcon]. The right cataract surgery occurred on day 119 after left cataract surgery. The patient was prophylactically prescribed ketorolac $0.4 \%$ eye drops q.i.d. and oral indomethacin $25 \mathrm{mg}$ t.d.s. both for 3 days. Both of these therapies were continued postoperatively in addition to chloramphenicol $0.5 \%$ and dexamethasone $0.1 \%$ eye drops both q.i.d.

Unfortunately, at day 32 postoperatively, BCVA remained poor at 6/18 in the right eye. OCT showed the presence of CME in the right eye (fig. 2b). No recurrence of CME was seen in the left eye at this time (fig. 1e). Topical dexamethasone $0.1 \%$ and prednisolone acetate $1 \%$ drops 6 times daily was used and gradually weaned over 4 months. Repeat OCT at 2.5 and 5 months showed complete resolution of CME (fig. 2c, d). While the patient continued to experience occasional flashes in both eyes, BCVA recovered to OD $6 / 6+4$ and OS $6 / 6+1$ five months after his second surgery. Improvement in his OS BCVA vision was noted up to 8 months after his initial operation.

\section{Discussion}

Bilateral cases of CME have been reported in association with systemic diseases including Crohn's disease [8] and infections [9]. Limited cases of bilateral CME after cataract surgery have been reported in the literature $[10,11]$.

Irvine-Gass syndrome following uncomplicated cataract surgery on this patient's first eye is not uncommon. The relatively unremarkable OCT scan obtained on day 9 postoperatively (fig. 1b), in the presence of symptoms, serves as a valuable reminder that evidence of CME on OCT examination may not appear until some weeks after cataract surgery. As such, the possibility of fresh macular pathology must be considered in the assessment of blur postoperatively, even in the light of recently performed normal OCT.

The occurrence of Irvine-Gass syndrome in this patient's second eye, despite topical and systemic prophylactic measures, was unexpected. His medical history contained no predisposing factors to developing CME. While it is probable that those who develop CME after their first cataract operation are at increased risk of developing it following surgery on their contralateral eye, there is a paucity of evidence for determining the specific level of risk in these individuals. Several studies have reported significant reductions in the incidence of clinical CME with prophylactic use of topical nonsteroidal anti-inflammatory drops, including ketorolac $0.4 \%$, and nepafenac $0.1 \%$, when used in conjunction with topical steroids or oral indomethacin, as was the case with this man's second eye [12-15]. Perhaps higher prophylactic therapy doses or the addition of preoperative topical indomethacin or steroids could be considered in similar cases. 
Shields et al:: A Case of Acute Bilateral Irvine-Gass Syndrome following Uncomplicated Phacoemulsification, Demonstrated with Optical Coherence Tomography

\section{Conclusion}

Irvine-Gass syndrome remains an important differential diagnosis in the evaluation of blurred vision after cataract surgery. A normal OCT scan 1 week after extraction does not preclude further CME, and repeated OCT studies may be warranted where unexplained blurred vision persists. Patients who develop CME following their first cataract operation should be counseled about the risks of developing the condition following surgery on the contralateral eye, irrespective of prophylactic measures.

\section{Statement of Ethics}

The patient's right and confidentiality have been well protected in all aspects, and he provided informed consent to publish prior to the authors submitting the article. All relevant ethical safeguards have been met in relation to patient protection.

\section{Disclosure Statement}

The authors have no financial interests or conflicts of interest.

\section{References}

1 Vukicevic M, Gin T, Al-Qureshi S: Prevalence of optical coherence tomography-diagnosed postoperative cystoid macular oedema in patients following uncomplicated phaco-emulsification cataract surgery. Clin Experiment Ophthalmol 2012;40:282-287.

2 Bringmann A, Reichenbach A, Wiedemann P: Pathomechanisms of cystoid macular edema. Ophthalmic Res 2004;36:241-249.

-3 Irvine SR: A newly defined vitreous syndrome following cataract surgery. Am J Ophthalmol 1953;36:599619.

-4 Henderson BA, Kim JY, Ament CS, Ferrufino-Ponce ZK, Grabowska A, Cremers SL: Clinical pseudophakic cystoid macular edema. Risk factors for development and duration after treatment. J Cataract Refract Surg 2007;33:1550-1558.

5 Loewenstein A, Zur D: Postsurgical cystoid macular edema. Dev Ophthalmol 2010;47:148-159.

-6 Lobo C: Pseudophakic cystoid macular edema. Ophthalmologica 2012;227:61-67.

7 Rotsos TG, Moschos MM: Cystoid macular edema. Clin Ophthalmol 2008;2:919-930.

-8 Mason J0 3rd: Bilateral phakic cystoid macular edema associated with Crohn's disease. South Med J 2002;95:1079-1080.

-9 Matuskova V, Vyslouzilova D: The possibility of the treatment of bilateral macular edema without the anti VEGF treatment - a case report (in Czech). Cesk Slov Oftalmol 2010;66:30-35.

-10 Scarpa G: Bilateral cystoid macular edema after cataract surgery resolved by vitrectomy. Eur J Ophthalmol 2011;21:677-679.

11 Chalioulias K, Tsaloumas M: Bilateral cystoid macular edema after phacoemulsification in post-laser in situ keratomileusis eyes. J Cataract Refract Surg 2007;33:1101-1103.

$\$ 12$ Wittpenn JR, Silverstein S, Heier J, et al: A randomized, masked comparison of topical ketorolac $0.4 \%$ plus steroid vs steroid alone in low-risk cataract surgery patients. Am J Ophthalmol 2008;146:554-560.

13 Yilmaz T, Cordero-Coma M, Gallagher MJ: Ketorolac therapy for the prevention of acute pseudophakic cystoid macular edema: a systematic review. Eye 2012;26:252-258.

14 Quintana NE, Allocco AR, Ponce JA, Magurno MG: Non steroidal anti-inflammatory drugs in the prevention of cystoid macular edema after uneventful cataract surgery. Clin Ophthalmol 2014;8:1209-1212.

15 Klein RM, Katzin HM, Yannuzzi LA: The effect of indomethacin pretreatment on aphakic cystoid macular edema. Am J Ophthalmol 1979;87:487-489. 


\section{Case Reports in \\ Ophthalmology}

\begin{tabular}{l|l}
\hline \multicolumn{2}{l}{ Case Rep Ophthalmol 2015;6:143-148 } \\
\hline DOI: 10.1159/000430087 & $\begin{array}{l}\text { @ 2015 S. Karger AG, Basel } \\
\text { www.karger.com/cop }\end{array}$ \\
\hline
\end{tabular}

Shields et al:: A Case of Acute Bilateral Irvine-Gass Syndrome following

Uncomplicated Phacoemulsification, Demonstrated with Optical Coherence Tomography

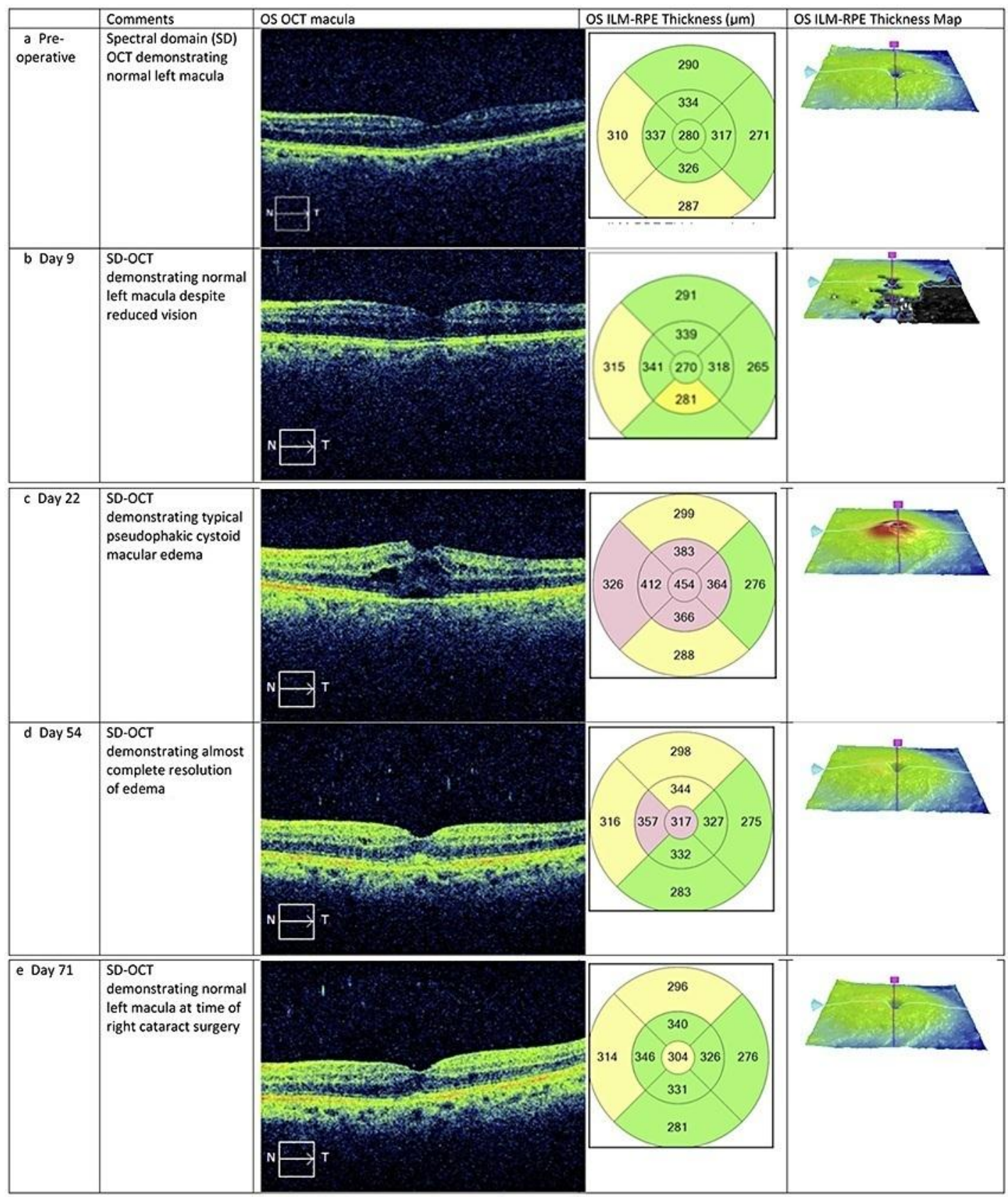

Fig. 1. OCT of the left macula. OS = Outer segment; ILM = internal limiting membrane; RPE = retinal pigment epithelium. 


\section{Case Reports in \\ Ophthalmology}

\begin{tabular}{l|l}
\hline \multicolumn{2}{l}{ Case Rep Ophthalmol 2015;6:143-148 } \\
\hline DOI: 10.1159/000430087 & $\begin{array}{l}\text { @ 2015 S. Karger AG, Basel } \\
\text { www.karger.com/cop }\end{array}$ \\
\hline
\end{tabular}

Shields et al.: A Case of Acute Bilateral Irvine-Gass Syndrome following Uncomplicated Phacoemulsification, Demonstrated with Optical Coherence Tomography

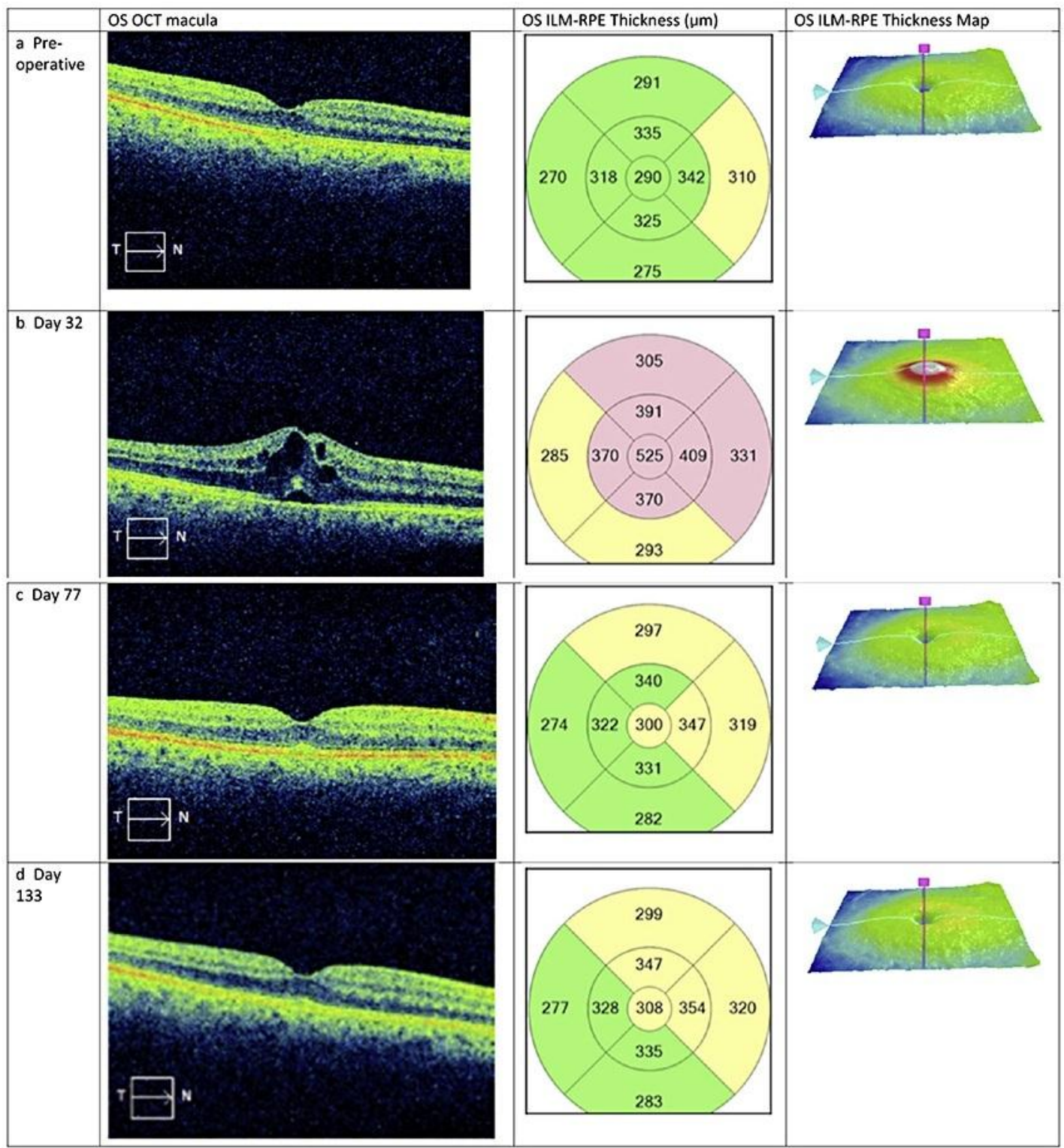

Fig. 2. OCT of the right macula. OS = Outer segment; ILM = internal limiting membrane; RPE = retinal pigment epithelium. 